\title{
Le emissioni odorigene degli impianti di depurazione delle acque reflue:
}

\section{campionamento, conservazione ed analisi}

\author{
Isidoro Silenzio ${ }^{1}$ \\ ${ }^{1}$ SEEDotti dall'Ambiente
}

\begin{abstract}
Una delle principali caratteristiche che si attribuisce all'odore è quella della soggettività. Misurare, quindi, la concentrazione di un odore all'interno di un contenitore chiuso può apparire un'impresa difficile. Esistono, però, da molti anni, tecniche e strumenti che consentono di determinare la quantità di odore presente all'interno di un campione di aria prelevato da una sorgente gassosa, liquida o addirittura solida. Molte di queste tecniche sono basate sull'uso dei nasi umani come strumento di misurazione. La maggior parte degli studi scientifici attuali affronta, proprio, il problema della soggettività: l'uso del naso umano, infatti, influenza la misurazione dell'odore perché entrano in gioco molteplici fattori, come lo stato di salute, l'età della persona, la provenienza geografica, le abitudini, etc. Ma esistono altri fattori di natura più tecnica che, spesso, vengono sottovalutati. Infatti, sperimentazioni scientifiche dimostrano che l'utilizzo di diversi dispositivi di campionamento e di contenitori costituiti da diverso materiale possono influenzare in maniera differente l'odore contenuto al loro interno. In questo studio vengono, infatti, descritti e confrontati gli effetti che le sacche di campionamento e di conservazione del campione di aria, costituite da tre diversi materiali plastici (Nalophan, Teflon e Tedlar), possono avere sullo stesso campione gassoso. La valutazione di questi effetti si basa sul confronto della concentrazione di odore di tali campioni misurata in diversi intervalli temporali intercorsi tra il momento del campionamento e l'analisi in laboratorio. I risultati mostrano che i tre materiali utilizzati per le sacche influenzano in maniera differente il campione di aria al loro interno. Questi risultati devono essere presi in considerazione per capire come e in quali condizioni utilizzarli, allo scopo di migliorare le tecniche e le tecnologie di misurazione dell'odore.
\end{abstract}




\section{La valutazione delle emissioni odorigene prove- nienti dagli impianti di depurazione}

Le emissioni odorigene che, la maggior parte delle volte, vengono prese a riferimento dagli studi scientifici, per la loro diretta connessione a problemi di natura sociale, sono quelle provenienti dagli impianti di trattamento delle acque reflue ${ }^{1} 2$. Infatti, la vicinanza a odori sgradevoli, come quelli degli impianti di depurazione, produce inevitabilmente un malessere fisico e psicologico, che si ripercuote sulla qualità della vita dei residenti e sulla svalutazione economica degli edifici e delle attività nei pressi dell'impianto ${ }^{3}$. Dato che l'esposizione prolungata ad un odore può risultare pericolosa per la salute umana con effetti da non sottovalutare ${ }^{45}$, risulta necessario valutare quantitativamente l'odore. L'odore viene valutato, sempre più spesso, mediante una modalità di misurazione, detta olfattometria dinamica, prevista dalla normativa europea EN 13725, che ha come obiettivo proprio la definizione di tale metodo 6 7. La normativa prevede che un gruppo di persone valuti l'odore dei campioni di gas raccolti in sacche di campionamento entro e non oltre 30 ore dal campionamento stesso. La valutazione di questi odori può essere più o meno soggettiva (entrano in gioco fattori fisiologici e psicologici) e in tal senso il metodo dell'olfattometria dinamica presenta, dunque, delle problematiche che devono essere tenute in considerazione ${ }^{8}$ 9 . Tra queste rientrano anche alcune di natura tecnica legate alle modalità e ai tempi di conservazione del campione gassoso ${ }^{10}$. Nel presente studio ${ }^{10}$ vengono presentati i risultati delle misurazioni di odore effettuate su alcuni campioni gassosi prelevati con sacche di tre materiali diversi (Nalophan, Teflon e Tedlar) da un impianto di depurazione delle acque reflue. In questi risultati si è messa in evidenza l'instabilità dell'odore nei diversi tipi di sacche al variare del tempo di permanenza del gas nelle sacche stesse, allo scopo di ottimizzare la metodologia prevista dalla normativa europea e rendere la misurazione più accurata e ripetibile nelle indagini future.

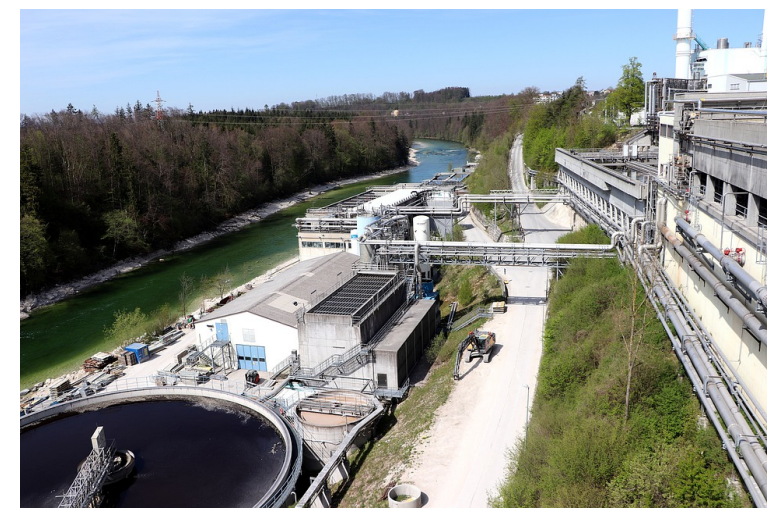

Figure 1: Impianto di depurazione delle acque reflue 


\section{Gli strumenti di campionamento e i risultati delle analisi olfattometriche}

La campagna di campionamento è stata eseguita in un impianto di depurazione a scala reale. II punto di prelievo dei campioni è rimasto costante per tutta la campagna ed è stato localizzato presso l'unità di trattamento dei fanghi da depurazione, ovvero la frazione più solida che viene allontanata dal liquido depurato. Allo scopo di evitare la contaminazione dell'aria prelevata, il campionamento è stato eseguito mediante la tecnica del "polmone". Tale tecnica prevede che la sacca di campionamento sia inserita in un involucro rigido; nell'intercapedine tra l'involucro e la sacca viene creato il vuoto mediante una pompa che aspira aria; questa operazione permette alla sacca di riempirsi del campione gassoso da analizzare. Le analisi olfattometriche sono state condotte da quattro persone specializzate presso la stessa sede in cui è localizzato l'impianto, utilizzando uno strumento standardizzato, detto olfattometro, con il semplice metodo del "sì/no". Questo metodo, presentato dalla già citata normativa europea, prevede che ogni persona indichi se percepisce o meno l'odore del gas, premendo un pulsante. Le sacche utilizzate per il campionamento avevano una capacità di 30 litri ed erano costituite da tre diversi materiali plastici (Nalophan, Teflon e Tedlar) per i quali variava semplicemente lo spessore. I campioni sono stati prelevati ogni giorno per 7 giorni per un totale di 21 campioni. Ogni campione è stato analizzato in laboratorio mediante olfattometria dinamica dopo $3,7,14,30$ e 40 ore dopo il campionamento per un totale di 105 concentrazioni di odore determinate.

I risultati mostrano che per tutte e tre le tipologie di sacche la concentrazione dell'odore diminuisce nell'arco di 14 ore dal campionamento, con un decadimento più marcato dopo le 30 ore (Figura 2). Ciò conferma che il valore delle 30 ore, così come indicato dalla normativa europea, risulta il limite superiore entro il quale effettuare le analisi olfattometriche.

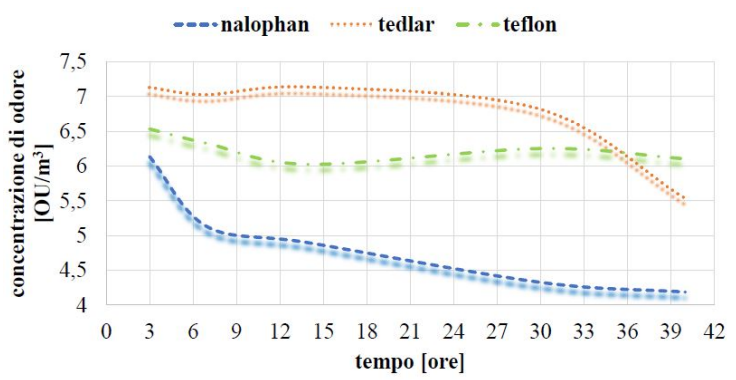

Figure 2: Esempio di variazione della concentrazione di odore nel tempo estratto dai risultati della campagna 1 (adattato da ${ }^{10}$ )

Concentrandosi principalmente sui materiali, dai risultati si nota che le sac- 
che in Nalophan e in Tedlar sono meno affidabili rispetto a quelle in Teflon, perché tendono a trattenere di meno l'odore. Come si può notare anche dalla Figura 2 la diminuzione della concentrazione di odore per le sacche in Nalophan e in Tedlar è maggiore (le curve pendono maggiormente) rispetto a quella delle sacche in Teflon. Infine, si è notato che per ogni misurazione della concentrazione di odore $\mathrm{i}$ sacchi in Tedlar presentano dei valori inziali sempre maggiori rispetto agli altri due, come si può vedere anche dalla Figura 2. Per quest'ultimo materiale alcuni studi dimostrano e confermano l'influenza sulla concentrazione del campione gassoso ivi contenuto. Uno studio di ${ }^{11}$, condotto su materiale agricolo, mostra che l'odore di fondo delle sacche in Tedlar produce una significativa distorsione dell'odore dopo 24 ore di stoccaggio del campione di aria al suo interno. Un secondo studio di ${ }^{12}$ mostra, invece, che per alcuni composti la permanenza del gas all'interno delle sacche in Tedlar produce nel tempo una diminuzione, anche del $100 \%$, della concentrazione e per altri, invece, un aumento.

\section{L'utilità dei risultati olfattometrici}

Analizzare l'odore mediante olfattometria dinamica è importante non solo per la misura dell'odore, ma anche per capire quali sono i fattori che influenzano l'analisi, come per esempio gli strumenti e i materiali utilizzati e il tempo di permanenza del gas nella sacca. II presente studio si limita all'analisi e discussione dei risultati ottenuti, senza esprimere giudizi sulle conseguenze che tali risultati possono determinare sulle strategie di gestione degli odori e sulle tecnologie di abbattimento degli stessi. I risultati ottenuti parlano chiaro: la concentrazione dell'odore nel tempo tende a diminuire, soprattutto dopo 30 ore dal campionamento, come indicato anche dalla normativa europea EN 13725. La conservazione nei sacchi in Teflon è più affidabile rispetto a quella nei sacchi in Nalophan e in Tedlar. Allo scopo di ottenere risultati affidabili e soprattutto ripetibili nelle misurazioni future, si è visto che le analisi olfattometriche vanno condotte sempre allo stesso intervallo e non oltre le 14 ore dal campionamento, poiché questo intervallo mostra delle diminuzioni di concentrazione di odore minori. Tale studio dovrà essere confermato nel futuro da ulteriori misurazioni, magari su altre sorgenti da cui proviene l'odore e che tengano conto anche di ulteriori fattori come il volume di aria prelevato, le modalità di conservazione e di trasporto dei campioni, in maniera da supportare la ricerca scientifica nello sviluppo di tecnologie per la misurazione dell'odore sempre più rapide e affidabili. 


\section{References}

1.Capelli, L., Sironi, S., Rosso, R. D. \& Céntola, P. Predicting odour emissions from wastewater treatment plants by means of odour emission factors. Water Research 43, 1977-1985 (2009).

2.Zarra, T., Reiser, M., Naddeo, V., Belgiorno, V. \& Kranert, M. Odour Emissions Characterization from Wastewater Treatment Plants by Different Measurement Methods. Chemical Engineering Transactions 40, 37-42 (2014).

3.Stellacci, P., Liberti, L., Notarnicola, M. \& Haas, C. N. Hygienic sustainability of site location of wastewater treatment plants. Desalination 253, 51-56 (2010).

4.Sironi, S., Capelli, L., Céntola, P., Rosso, R. D. \& Pierucci, S. Odour impact assessment by means of dynamic olfactometry dispersion modelling and social participation. Atmospheric Environment 44, 354-360 (2010).

5.Devai, I. \& DeLaune, R. D. Emission of Reduced Malodorous Sulfur Gases from Wastewater Treatment Plants. Water Environment Research 71, 203-208 (1999).

6. Frechen, F.-B. Odour emission inventory of German wastewater treatment plants - odour flow rates and odour emission capacity. Water Science and Technology 50, 139-146 (2004).

7.Capelli, L., Sironi, S. \& Rosso, R. D. Odor Sampling: Techniques and Strategies for the Estimation of Odor Emission Rates from Different Source Types. Sensors 13, 938-955 (2013).

8.Gostelow, P., Parsons, S. A. \& Stuetz, R. M. Odour measurements for sewage treatment works. Water Research 35, 579-597 (2001).

9.Zarra, T., Naddeo, V., Belgiorno, V., Reiser, M. \& Kranert, M. Odour monitoring of small wastewater treatment plant located in sensitive environment. Water Science and Technology 58, 89-94 (2008).

10.Zarra, T., Reiser, M., Naddeo, V., Belgiorno, V. \& Kranert, M. A comparative and Critical Evaluation of Different Sampling Materials in the Measurement of Odour Concentration by Dynamic Olfactometry. Chemical Engineering Transactions 30, 307-312 (2012).

11.Trabue, S. L., Anhalt, J. C. \& Zahn, J. A. Bias of Tedlar Bags in the Measurement of Agricultural Odorants. Journal of Environment Quality 35, 1668 (2006).

12.Szyłak-Szydłowski, M. Odour Samples Degradation During Detention

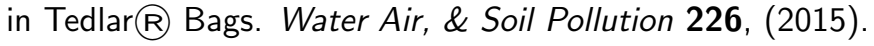

